\title{
CORRIGENDUM
}

\section{Stabilisation and drag reduction of pipe flows by flattening the base profile - CORRIGENDUM}

\author{
Elena Marensi, Ashley P. Willis and Rich R. Kerswell \\ doi:10.1017/jfm.2018.1012, Published by Cambridge University Press, \\ 28 January 2019
}

Marensi, Willis \& Kerswell (2019) has an error in table 3 and in the lower graph of figure 11 because the contribution from the pressure drop across the baffle was not included in the drag reduction results. Throughout the paper, 'drag reduction' should be read as 'frictional drag reduction'. All the calculations were otherwise correct and values for the full drag reduction are reported in the following.

The force balance in the streamwise direction is

$$
\beta(t)=\underbrace{-\left.\frac{1}{2} \frac{\overline{\partial \tilde{w}}^{\theta, z}}{\partial r}\right|_{r=1}}_{\mathscr{T}_{w}}+\underbrace{\frac{\operatorname{Re}}{2} \int_{0}^{1} \overline{-\boldsymbol{F} \cdot \hat{z}^{\theta, z} r \mathrm{~d} r}}_{\mathscr{B}},
$$

where $\overline{(\bullet)}^{\theta, z}$ indicates cylindrical-surface average, $\mathscr{T}_{w}$ is the perturbation shear stress at the wall and $\mathscr{B}$ is the extra drag due to the local pressure drop across the baffle. The energy balance is

$$
\underbrace{\left\langle\boldsymbol{u}_{t o t} \cdot \nabla p_{t o t}\right\rangle}_{\mathcal{I}}=\underbrace{\frac{1}{\operatorname{Re}}\left\langle\left(\nabla \times \boldsymbol{u}_{t o t}\right)^{2}\right\rangle}_{\mathcal{D}}+\underbrace{\left\langle-\boldsymbol{F} \cdot \boldsymbol{u}_{t o t}\right\rangle}_{\mathcal{P}},
$$

where $\langle(\bullet)\rangle$ indicates volume integral, $p_{\text {tot }}$ is the total pressure field, $\mathcal{I}=(2 \pi L / R e)$ $(1+\beta)$ is the input energy needed to drive the flow, $\mathcal{D}$ is the viscous dissipation and $\mathcal{P}$ is the work done by the drag force $\boldsymbol{F}(\boldsymbol{x}, t)$ against the flow. The input energy relative to that for laminar flow is given by

$$
(1+\beta)_{\text {lam } / \text { turb }}^{A}=\frac{\mathcal{I}_{\text {lam } / \text { turb }}^{A}}{\mathcal{I}_{\text {lam }}} .
$$

Here, the superscript ' $A$ ' indicates the forced case $(A>0)$, no superscript indicates the unforced case and the subscript 'lam' or 'turb' refers to the flow being laminar $\left(E_{0}<E_{c}\right)$ or turbulent $\left(E_{0}>E_{c}\right)$ at the current value of $A \geqslant 0$. The figures of $(1+\beta)$ reported in table 3 of Marensi et al. (2019) did not include the quantity $\mathscr{B}$, corresponding to $\mathcal{P}$.

The corrected values of $(1+\beta)_{\text {lam }}^{A=0.005}$ are now reported in the third column of table 1. Equation (3.7) should be corrected to

$$
\begin{aligned}
\mathcal{D R}_{\text {lam } / \text { turb }} & :=\frac{\mathcal{I}_{\text {turb }}-\mathcal{I}_{\text {lam } / \text { turb }}^{A}}{\mathcal{I}_{\text {turb }}}=\frac{\mathcal{D}_{\text {turb }}-(\mathcal{D}+\mathcal{P})_{\text {lam } / \text { turb }}^{A}}{\mathcal{D}_{\text {turb }}} \\
& =\frac{(1+\beta)_{\text {turb }}-(1+\beta)_{\text {lam } / \text { turb }}^{A}}{(1+\beta)_{\text {turb }}}
\end{aligned}
$$




$\begin{array}{rccccc}\text { Re } & (1+\beta)_{\text {turb }} & (1+\beta)_{\text {lam }}^{A=0.005} & \mathcal{D R}_{\text {lam }}(\%) & \left(1+\mathscr{T}_{w}\right)_{\text {lam }}^{A=0.005} & \mathcal{F \mathcal { R }}_{\text {lam }}(\%) \\ 2400 & 1.695 & 1.59 & 6 & 1.143 & 32.5 \\ 3500 & 2.250 & 1.87 & 17 & 1.202 & 46.5 \\ 5000 & 2.940 & 2.2 & 25 & 1.275 & 56.6 \\ 7000 & 3.783 & 2.68 & 29 & 1.354 & 64.2 \\ 10000 & 4.944 & 3.35 & 32 & 1.48 & 70\end{array}$

TABLE 1. (Corrigendum of table 3 in Marensi et al. (2019)). Effect of a baffle of amplitude $A=0.005$ on the nonlinear stability of the laminar state at different Reynolds numbers in a $L=5 D$ pipe. Disturbances that are just above the laminar-turbulent boundary in the unforced case, i.e. just capable of triggering turbulence in the unforced case, are used as initial conditions.

The corrected values of $\mathcal{D} \mathcal{R}_{\text {lam }}$ are now reported in the fourth column of table 1 . A lower, but still significant, drag reduction is obtained, compared to the figures previously presented. Starting from initial disturbances just above the laminarturbulent boundary in the unforced case, indeed, only a small amplitude $A$ is needed to avoid turbulence being triggered. As the initial perturbation energy is increased, $A$ needs to be increased to keep the flow laminar, as shown in $\S 3.3$ of Marensi et al. (2019), and the extra drag due to the baffle also increases, as discussed later.

The values reported in the last two columns of table 3 in Marensi et al. (2019) are still quantities of interest. The baffle, indeed, modifies the mean streamwise velocity profile $W_{m}(r)=\left(1-r^{2}\right)+\overline{\tilde{w}}^{\theta, z}$ and thus the shear stress $\mathcal{S}$ at the wall, relative to the unforced laminar value $\mathcal{S}_{\text {lam }}$, namely

$$
\frac{\mathcal{S}}{\mathcal{S}_{\text {lam }}}:=\frac{\left(\partial_{r} W_{m}\right)_{\mid r=1}}{-2}=1+\mathscr{T}_{w} \quad\left(\text { with } \partial_{r}=\partial / \partial r\right),
$$

is also modified. The latter is a measure of the resistance encountered by the flow at the wall. For a baffle of amplitude $A=0.005$, the values of $\mathcal{S}_{\text {lam }}^{A=0.005} / \mathcal{S}_{\text {lam }}=(1+$ $\left.\mathscr{T}_{w}\right)_{\text {lam }}^{A=0.005}$ were reported in the third column of table 3 in Marensi et al. (2019) (now fifth column of table 1). The corresponding frictional drag reduction is defined as

$$
\mathcal{F} \mathcal{R}_{\text {lam } / \text { turb }}:=\frac{\mathcal{S}_{\text {turb }}-\mathcal{S}_{\text {lam } / \text { turb }}^{A}}{\mathcal{S}_{\text {turb }}}=\frac{\left(1+\mathscr{T}_{w}\right)_{\text {turb }}-\left(1+\mathscr{T}_{w}\right)_{\text {lam } / \text { turb }}^{A}}{\left(1+\mathscr{T}_{w}\right)_{\text {turb }}},
$$

and was reported in the last column of table 3 in Marensi et al. (2019) (now last column of table 1).

The labels on the vertical axes of the bottom graph of figure 11 of Marensi et al. (2019) should $\operatorname{read} \mathcal{S} / \mathcal{S}_{\text {lam }}$, on the left, and $\mathcal{F} \mathcal{R}(\%)$, on the right (see figure 1 ). The rest remains unchanged, including the ensuing discussion at pp. 869-870 upon substitution of $\mathcal{D} \mathcal{R}$ with $\mathcal{F} \mathcal{R}$.

The last sentence of p. 870 should read: 'The forcing is found to be beneficial up to $A_{c} \approx 0.008$ (and not $A_{c}=0.073$ ) where $\mathcal{D} \mathcal{R}_{\text {lam }}$ becomes negative.... The value of $A_{c}$ should also be corrected in the last paragraph of the conclusions. Indeed, at $A=0.01$, the corrected $(1+\beta)_{\text {lam }}^{A=0.01}=3.36$ is already higher than $(1+\beta)_{\text {turb }}=2.94$ in the unforced case and $\beta$ increases approximately linearly with $A$. At $A=0.03$, where a full collapse of turbulence is achieved and the laminar state remains the only global attractor, $(1+\beta)_{\text {lam }}^{A=0.03}=7.73$. However, assuming the flow remains laminar 


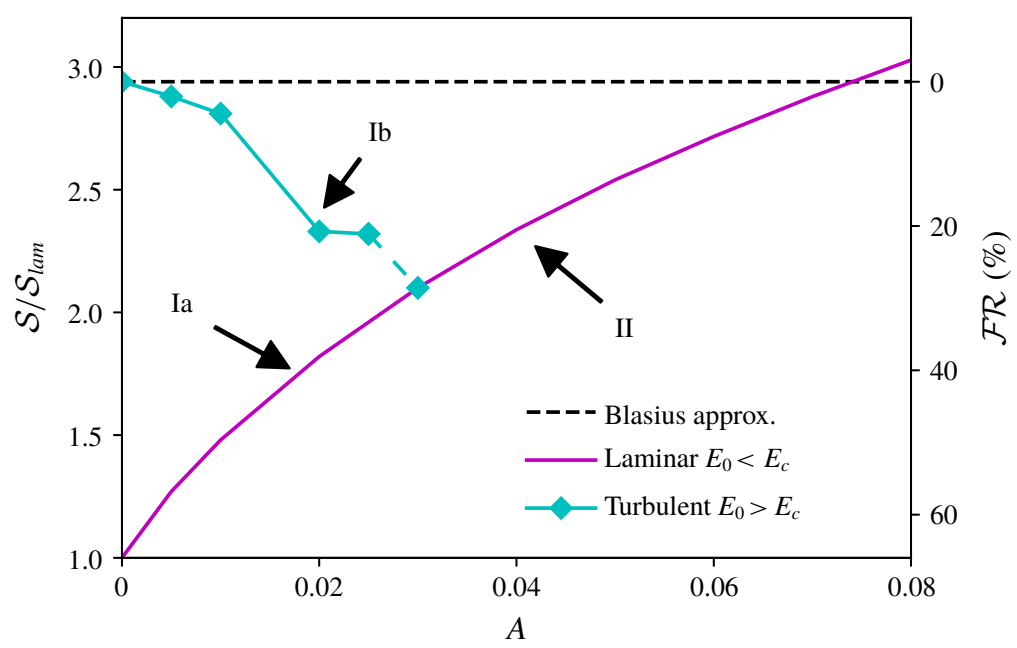

FIGURE 1. (Corrigendum of bottom graph of figure 11 in Marensi et al. (2019)). Effect of the baffle for different $A$ at $R e=5000$ : wall shear stress and skin-friction drag reductions versus the amplitude. For $A<0.03$ either laminar (Ia: $E_{0}<E_{c}$ ) or turbulent (Ib: $E_{0}>E_{c}$ ) skin-friction drag reductions are possible, for $A \geqslant 0.03$ turbulence is suppressed and the laminar state remains the only global attractor.

downstream of the baffle (or of the periodic array of baffles), a net energy saving will be achieved at a distance from the baffle where the shear stress reduction at the wall due to the relaminarised flow becomes larger than the extra drag caused by the baffle. For example, at $A=0.03$ and $R e=5000$ for a $5 D$ long pipe, we estimate that it takes $\sim 50 D$ to relaminarise the flow, i.e. the flow passes through the baffle $\sim 10$ times. A laminar section of flow of $\sim 200 D$ is then needed for the wall stress reduction to compensate for the baffle drag. The resulting break-even distance where $\mathcal{I}_{\text {lam }}^{A}=\mathcal{I}_{\text {turb }}$ is $L_{\text {even }} \approx 250 D$, consistent with the experiments of Kühnen et al. (2018). Note that the initial transient in the unforced trajectory is not taken into account in the above estimate and the (averaged) statistically steady turbulent value of $(1+\beta)_{\text {turb }}=2.94$ is instead considered for the whole distance $L_{\text {even }}$.

\section{Acknowledgements}

We would like to thank Dr Z. Ding for helping to identify the above error and Dr P. Ricco for insightful discussion.

\section{REFERENCES}

KÜhnen, J., Scarselli, D., Schaner, M. \& Hof, B. 2018 Relaminarization by steady modification of the streamwise velocity profile in a pipe. Flow Turbul. Combust. 100 (4), 919-943.

Marensi, E., Willis, A. P. \& Kerswell, R. R. 2019 Stabilisation and drag reduction of pipe flows by flattening the base profile. J. Fluid Mech. 863, 850-875. 